\title{
Nuevos medios, nuevos perfiles y conectividad emocional con la audiencia: los periodistas en la praxis profesional
}

\author{
Hada M. SÁnchez GonZALES y Sandra MÉndez Muros* \\ misago@us.es \\ sanmenmur@us.es
}

\begin{abstract}
(Abstracts y palabras clave al final del artículo)
Enviado: 8 de octubre de 2012

Evaluado: 20 de marzo de 2013

Aceptado: 6 de abril de 2013
\end{abstract}

\section{EL PERIODISTA Y LA AUDIENCIA: PERFILES 2.0}

La crisis económica ha afectado a la sociedad y amenaza al periodismo español. El periodista se ha visto aquejado porque se han producido un sin número de despidos en los medios de comunicación, reducción de salarios y, en algunos casos, pérdida de los derechos laborales e, incluso, algunas empresas periodísticas se han visto obligados a cerrar, lo que significa que muchos periodistas han ido al paro. La tasa de desempleo, según el Informe anual de la profesión periodística 2011 (APM, 2011), aumentó el $78 \%$ respecto al 2010, hasta alcanzar un total de 9.937 parados. Datos que indican que sólo uno de cada dos licenciados en Periodismo encuentra trabajo. No obstante, el primordial problema que argumentan los profesionales encuestados es la falta de independencia, así lo corrobora un $43,6 \%$. Otro dato que añadieron es la precariedad laboral.

En 2012, los datos arrojan que en el último año, un total de 1.667 puestos de trabajo han sido destruidos en el sector de los medios de comunicación: "Cierra Público con 164 empleados, $A D N$ baja la persiana con 60, Unión Editorial prepara alrededor de 200 bajas en El Mundo, el grupo Prisa espera concluir su Plan de Eficiencia operativa con el recorte de 448 puestos en el primer trimestre del año, varios grupos de comunicación rozan la quiebra y los rumores de recortes ensombrecen las empresas públicas" (Molina, 2012: 8).

\footnotetext{
* Hada M. Sánchez Gonzales es doctora en Periodismo, profesora adscrita al Departamento de Periodismo II de la Universidad de Sevilla. Coordinadora del Equipo de Investigación de Análisis y Técnica de la Información (HUM-212). Coordinadora académica de su Programa de Título Propio sobre "Experto Universitario en Periodismo Local, Medio Ambiente y Sociedad Civil e Innovación" de la Universidad de Sevilla. Apasionada por la tecnología, la innovación educativa, medios sociales y la emotividad en la Red desde la recepción del mensaje. Sandra Méndez Muros es doctora en Periodismo, profesora de la Facultad de Comunicación de la Universidad de Sevilla y miembro del Equipo de Investigación Análisis y Técnica de la Información.
} 
Dentro de este contexto desesperanzador y decepcionante, las tecnologías cobran especial protagonismo y el futuro del periodismo tiene que ver con Internet y los nuevos espacios de la Web social porque han pasado a formar parte de nuestra sociedad en casi todas las esferas de la vida, facilitando la comunicación, la información e interactuación del ciudadano. Los medios sociales han adquirido un papel protagónico en la sociedad como parte esencial del entorno enmarcado por nuevas formas de trabajar e, incluso, hasta de convivir.

No es difícil apreciar dentro del contexto social y tecnológico la expansión de la mente de los individuos en redes de acción recíproca sujetas a la tecnología de la microelectrónica. Y, es que la sociedad en Red emergió como "una nueva forma de organización social de la actividad humana en el último tramo del siglo XX" (Castells, 2006: 32 y 39) y ha quedado tan suficientemente asentada que permite integrar las herramientas digitales como mecanismos de desarrollo y fortalecimiento de la sociedad y, por consiguiente, del ciudadano.

Los hábitos de la audiencia han cambiado de parámetros, tal es así que el uso de los medios sociales es cada vez mayor por los internautas. Los datos más sobresalientes de lo que sucede en el ciberespacio en 60 segundos, según el estudio realizado por la consultoría de mercadeo Social Jumpstart, nos indican que los usuarios realizan " 175.000 tweets en promedio por minuto, que en Foursquare solamente se hacen 2.000 'checkins', y tal vez la más reveladora: que mientras en Facebook se envían 700.000 mensajes, en YouTube se ven 2 millones de videos. Casi el triple de mensajes en Facebook, aunque hay que tener en cuenta que cada acción requiere un esfuerzo y actitud distinta".

Los medios sociales han posibilitado la comunicación multidireccional y la identificación del ciudadano con su problemática socioeconómica y política, con sus intereses individuales y de grupo. Son la prueba palpable de democratización emocional de la información. El periodista no sólo tiene que enfrentarse a la crisis del periodismo, sino que tiene que adaptarse al nuevo entorno de comunicación frente a una audiencia más implicada y sensible ante los hechos.

El periodismo no puede ni debe mantenerse al margen de los medios sociales que emergen en Internet. Los periodistas tradicionales deben ser conscientes del nuevo papel que han de adoptar ante un público cada vez más activo y consciente de sí mismo en un mundo digitalizado. Asistimos a un panorama que propende que los periodistas asuman nuevos roles devenidos por la incursión de nuevos formatos debido a la convergencia mediática y de audiencias. Para ello, el periodista debe trabajar en dos variantes: competencias tecnológicas y temáticas de la mano de los valores éticos de la profesión.

El periodista tradicional debe adquirir las destrezas tecnológicas y temáticas, concibiendo al mismo tiempo que el periodismo debe ser conversación, por lo que es necesario que se instruyan en las herramientas que propician la Web 2.0. Periodistas como Ana Alfageme, de El Pais, expone que ha tenido claro desde un inicio que Internet y los medios sociales son la clave para el periodismo. Incluso, antes de incorporarse a su puesto como responsable de Medios Sociales, hizo lo que pudo para transmitir en directo noticias útiles y de interés para la audiencia. $\mathrm{La}$ periodista enfatiza que es necesario "llevar los valores del periodismo a los medios 
sociales, necesitamos, en general, que reporteros curtidos se involucren. Es importante conocer estos medios, su "modus operandi", pero también, y sobre todo, tener algo que contar" (Vargas, 2010).

La audiencia es lo verdaderamente importante en esta nueva cruzada informativa, comunicativa, social y tecnológica, por consiguiente, es necesario saber cuáles son sus necesidades y tener un plan para llegar al usuario y los procedimientos para medir la interactuación. En algunos medios, como El País, se procura que los periodistas, que aún no están familiarizados con la interacción con los lectores, "entren a esos foros, para aprender, para buscar fuentes, para ayudarse, para reforzar su marca personal. Y, en eso Eskup está siendo crucial. Es como meter dentro de casa algo que hay que salir a buscar fuera" (Vargas, 2010).

Sin duda alguna, los nuevos desafíos exigen cambios significativos en el trabajo del periodista y en la relación con los medios y la audiencia sin dejar de lado la ética y la calidad informativa. Los contenidos digitales exigirán en ciertos casos "una nueva forma de narrar, aunque siempre sin menoscabo de la calidad, que es la mejor garantía de supervivencia de nuestra profesión" (San Martín, 2012: 7).

Al periodista se le insta a que sea polivalente y que se adapte a las nuevas circunstancias del contexto actual, propiciado por mutaciones tecnológicas, el incremento de herramientas de producción y consumo de informaciones (López y Freire, 2010) y el desarrollo de competencias y habilidades tecnológicas. Tanto es así que, como dice el refrán, "un buen reportero es bueno en cualquier parte" ya no es convincente (Meyer, 2007: 10). Esta situación pone a los periodistas en alerta en relación a la oferta laboral y, por otra parte, en atención con la adquisición de nuevas destrezas tecnológicas y temáticas porque revertirá en una mayor demanda en la consecución de un puesto de trabajo.

Como dice Gillmor (2012), es posible que se trate de la época dorada del periodismo porque los periodistas pueden reinventar el periodismo con nuevas narrativas y ejecución de tareas profesionales relacionadas con el mundo 2.0 variando la redacción hacia un diálogo social más comprometido con la audiencia y, por otro lado, es factible hablar de un periodista multiuso con más funciones en la redacción para lograr una mayor productividad de la empresa y a menor costo. Debates aparte, en cualquier caso, se trata de nuevos espacios que ha propiciado la incursión de nuevos perfiles para la ejecución de dichas labores.

La demanda de nuevas figuras profesionales es indiscutible debido a que las empresas y emprendedores solicitan nuevos quehaceres en la Web social. Cabe citar las tres figuras periodísticas más utilizadas por los medios de comunicación españoles: a) Editor de Medios Sociales, «Social Media Editor o Content Manager», b) «Community Manager» y c) Director de Redes Sociales, «Social Media Manager» o «Social Media Director». Cada puesto es desempeñado según las necesidades de la empresa periodística. También, es posible percibir dos o tres figuras profesionales en un mismo medio o grupo de comunicación o, en su caso, que una persona haga la función de cada una de las tres figuras 2.0 bajo demanda.

Es plausible citar casos concretos de empresas periodísticas españolas que han incorporado a sus redacciones las nuevas figuras profesionales. En el primer caso, señalamos a la periodista Ana Alfageme, responsable de Medios Sociales del $E l$ 
País, quien tiene como función propulsar la intervención en redes sociales de sus lectores, y que manifiesta que los medios sociales son un medio de comunicación más y "constituyen una voz alternativa y espontánea: miles de personas alzando su mensaje, en comunión o diferenciándose y, en ocasiones, son más ágiles (por ejemplo, Twitter) que las propias cabeceras informativas" (Vargas, 2010).

En el segundo caso, cabe resaltar la función de profesionales del periodismo que trabajan como «Community Manager», por ejemplo, el periodista Ángel de los Ríos, de Diario Sur. Su actividad medular se centra en saber qué quiere comunicar el medio de comunicación y luego trasladársela al usuario, con el fin de establecer un contacto directo entre ambos. Además, monitoriza y fomenta la participación en las redes sociales con el criterio de buscar nuevos líderes.

Citaremos en el tercer caso la figura de Director de Redes Sociales, de la mano del periodista Ramón Puchades de Unidad Editorial. Entre sus funciones define y coordina la estrategia social y digital de las diferentes cabeceras del grupo ( $E l$ Mundo, Marca, Expansión, Telva y Diario Médico) porque se trata de un escenario donde las audiencias están fragmentadas, como reconoce al señalar que "cuanto más potente sea su marca personal, en conjunción con la marca corporativa, más potente es el conjunto... Ya no le avala tanto el número de artículos, sino la gente que confía en él a la hora de leer esos artículos" (Herreros, 2012).

Las empresas periodísticas han apostado por el uso de los medios sociales en la redacción y han elaborado incluso sus propias guías de estilo con normas específicas para redactar y usar los medios sociales. Podemos señalar el ejemplo de la agencia $E f e$ en España y de medios de comunicación en el extranjero como: $B B C$, The Washigton Post, Los Angeles Time y la agencia Reuters.

En definitiva, el periodista está expuesto a la ley de la oferta y la demanda de la empresa de comunicación siempre y cuando decida que será un profesional dependiente de un medio, a diferencia de innovar y emprender con un producto singular que ofrezca un valor añadido a la sociedad. En cualquier caso, su única salida es su credibilidad con respecto a la audiencia, por lo tanto, podríamos decir que el profesional del periodismo se convertiría en marca pública (según la empresa) y privada.

\section{EL USO DE LOS MEDIOS SOCIALES: CONEXIÓN EMOCIONAL}

Internet ha posibilitado la innovación y convergencia tecnológica transgrediendo en tiempo y espacio la forma de dar a conocer una noticia, por ello, nos planteamos la siguiente interrogante: ¿qué aplicaciones trae consigo los medios sociales para la labor del periodista?

Los medios sociales tienen unas particularidades propias que permiten estrechar la relación entre el periodista y la fuente de información. Podríamos decir que es una táctica caracterizada por la ausencia de la cercanía física entre el periodista y la fuente que permiten establecer un contacto directo con las personas y aportar un panorama diferente a la información publicada asiduamente en los medios. Es factible hablar de casos concretos de cobertura informativa como, por ejemplo, el tiroteo en 
la base militar texana "Fort Hood" en Estados Unidos, el terremoto de Haití y de Chile, el Mundial de Sudáfrica 2010 y el caso de Oriente Medio, entre otros.

De los casos nombrados, abordaremos el conflicto en el Oriente Medio (revueltas de Egipto). Los blogs, YouTube, Facebook, Bambuser, Twitter, etc. fueron los canales protagonistas que dieron a conocer lo que estaba pasando en El Cairo. Ello generó una repercusión sin precedentes; las herramientas virtuales se convirtieron en instrumentos de difusión. Podemos nombrar algunos periodistas que utilizaron estos canales sociales de comunicación pera difundir la información a nivel mundial como, por ejemplo, Wael Abbas, gestor de un blog especializado sobre la revuelta de El Cairo y el abuso del ex presidente de Egipto Hosni Mubarak a la población. El periodista también difundió la noticia a través de Twitter con el nombre@waelabbas. También, podemos citar a la periodista Nora Younis (@norayounis), quien publicó la información a través de su blog y vía Twitter.

Igualmente, medios como Al Jazeera han sabido aprovechar los recursos de los medios sociales para dar a conocer la revuelta del pueblo egipcio. La cadena qatarí está presente en YouTube, Facebook y en Twitter, lo que ha provocado una mayor difusión de la movilización democrática de los ciudadanos, convirtiéndose en uno de los medios de referencia en la cobertura de la revolución de los jóvenes de Egipto. Es más, según Riyaad Minty, responsable de Medios Sociales de Al Jazeera (Lajas, 2011), la cadena era escuchada por ciudadanos estadounidenses en la emisión en directo vía online, tal es así que casi el 50\% de la audiencia era de Estados Unidos.

Los medios sociales han superado a los periódicos como fuentes de información en los países árabes, de acuerdo con el estudio del Centro Internacional de Asistencia a los Medios de Comunicación (CIMA), el 50\% de la población egipcia se informa mediante Internet, frente a un $34 \%$ que lo hace concibiendo los medios tradicionales (prensa escrita) (Ghannam, 2011). De igual forma, la consultoría Wandren PD (2011) efectuó una encuesta sobre el uso de Twitter en las protestas de Oriente Medio y para lo cual utilizaron una muestra de tres millones de tweets. De los resultados se desprende que los hashtags más utilizados en el conflicto de El Cairo fueron \#Egypt y \#Libya.

Sin lugar a dudas, los medios sociales cobran protagonismo con la difusión de la información sobre las revueltas de Egipto y, asimismo, con la emisión de imágenes y vídeos que dieron la vuelta al mundo y que dejaron en evidencia el tipo de gobierno de Mubarak durante más de 30 años en el poder. En YouTube se subieron en menos de 24 horas 3.470 vídeos referidos a Egipto y 2.740 a Mubarak, según el estudio del CIMA (Ghannam, 2011).

Visto el panorama, las empresas periodísticas españolas han incorporado fórmulas para adherir a la audiencia y para estar presente en los entornos sociales 2.0. El Informe sobre Medios de Comunicación en Redes Sociales en España (GAD, 2011) ratifica la presencia de la prensa en Twitter con 1.700 .000 seguidores habituales. Igualmente, las cadenas de televisión han visto una nueva posibilidad en YouTube, por ello, han volcado sus contenidos consiguiendo 165.000 suscriptores fijos. De igual forma, las emisoras de radio no se podían quedar atrás; están presentes en Facebook con 1.200.000 seguidores porque les ayuda a mantener la participación de los usuarios que tienen la misma afición al programa emitido. 
Si bien es cierto que el periodista no debe abandonar sus fuentes tradicionales, al mismo tiempo tampoco debe rechazar la que le brindan los medios 2.0 porque son "fuentes informativas alternativas" próximas al hecho y sin restricción alguna (Maciá, 2007). Indudablemente, los medios sociales se constituyen en un reto para el periodista en la búsqueda de la información útil y fidedigna de interés para el ciudadano. Además, de cara a los próximos Juegos Olímpicos de Londres 2012, algunos periodistas y autores ya han publicado una recopilación de fuentes para cubrir la información (Orihuela, 2012):

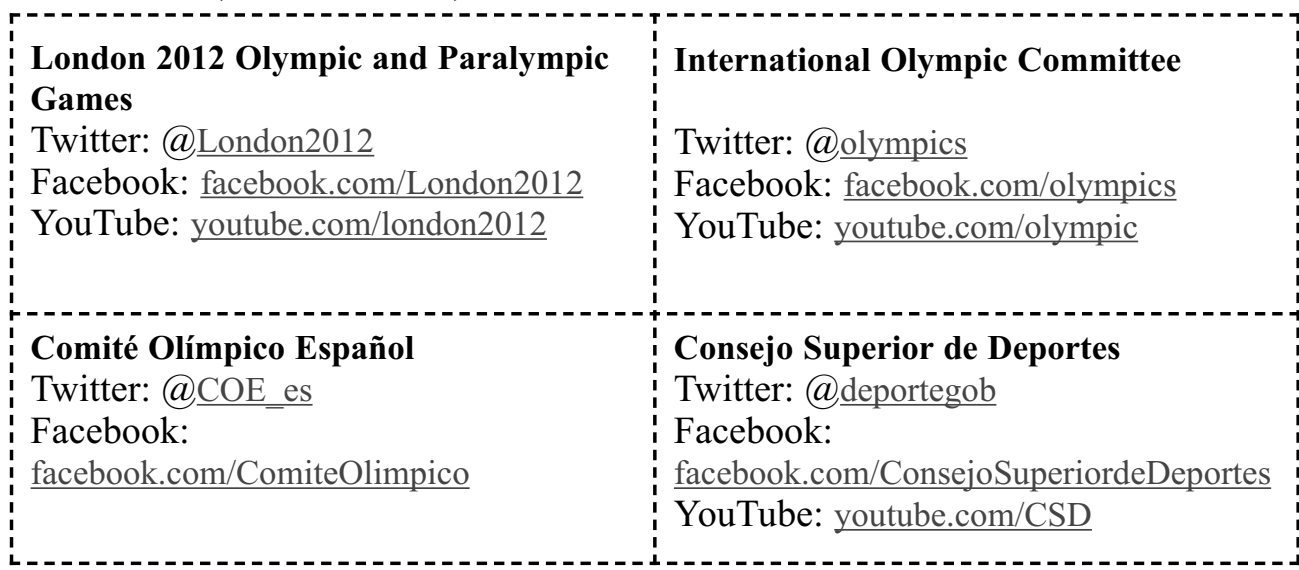

Es perceptible que Twitter, Facebook y YouTube son los medios sociales que cobran mayor protagonismo por encima del resto como fuentes de información; en este caso concreto, para la información que se difundirá a nivel mundial sobre los Juegos Olímpicos de Londres 2012.

Las herramientas sociales contribuyen, además, al desarrollo de las competencias emocionales en la labor del periodista. Asimismo, no es difícil percibir la conectividad emocional en la audiencia y más aún es estos entornos ya que "nuestras acciones dependen de nuestras emociones". Consumimos a partir del "significado y no de la necesidad asociada a las tecnologías como principal expresión del contexto actual, de ahí que no es de extrañar que la relación de los individuos es provocada por el significado. Construimos nuestras realidades como seres humanos sociales donde las emociones cambiarían de sentido según el significado que le demos dentro del contexto de interactuación" (Sánchez, 2012).

Podemos citar un caso real en el que se ha establecido la conexión emocional con la audiencia a través de los medios sociales en las revueltas de Oriente Medio, es decir, cuando los medios sociales fueron los protagonistas de la información que se difundía en El Cairo. Uno de los ejemplos resaltantes que podríamos nombrar es la crónica periodística que, por medio de la sucesión de mensajes cortos, se publicaron en el microbloggin Twitter por el periodista Mohamed Abdelfattah con el nombre del usuario (@mfatta7) y con fecha 25 de enero de 2011) (RIA Novosti, 2011):

Gas lacrimógeno (21:12 hrs.);

Me asfixio (21:13 hrs.); 
¡Socorro! (21:24 hrs.);

Me dieron fuerte paliza (21:27 hrs.);

Me van a detener....

No cabe duda de la conectividad emocional que estas plataformas establecen con la audiencia. De ahí que no es nada raro el éxito de los medios sociales, más aún, de sus aplicaciones y de la actitud empática que se revela en la web 2.0. Además, cabe añadir que son espacios que reducen el estrés, según Paul Zak, y aumentan la percepción de cercanía con la comunidad debido a la oxitocina, hormona que actúa como neurotransmisor en el seso: "El cerebro parece ver la interacción virtual como si las personas estuvieran junto a nosotros" (Zak, 2010).

Las empresas periodísticas deben seguir la lógica del diálogo y participación próxima a la audiencia, más aún cuando el escenario lo propicia y concede especial protagonismo al ciudadano; debido a ello, nos interesa conocer cuál es la opinión de los periodistas de dos medios locales sobre las herramientas sociales 2.0 y determinar el uso que realizan en la profesión.

\section{METODOLOGÍA}

Nuestro principal objetivo es conocer la opinión de los periodistas que trabajan actualmente con herramientas comunicativas de la Web 2.0 para calibrar su utilidad real en la praxis profesional, así como los usos que le dan en términos de conectividad social y emocional. Partimos, para ello, de la hipótesis de que en el entorno tecnológico actual los periodistas locales en ejercicio no sólo conocen las herramientas y medios sociales, sino que los usan con asiduidad para la configuración de un periodismo de calidad, si bien mantienen hasta el momento un grado de utilización bajo con respecto al potencial que se advierte en periodistas extranjeros que han cubierto acontecimientos internacionales.

Metodológicamente, estamos ante un estudio de prevalencia y de naturaleza descriptiva, cuyo fin es dar cuenta de las relaciones entre variables sin establecer vínculos de causa-efecto. Se trata de un análisis univariable con técnicas cualitativas (establecimiento de categorías: motivaciones, frecuencias, conceptos y credibilidad) y cuantitativas (estadísticas), del que se extraen resultados en porcentajes. Aplicamos la estadística de forma descriptiva, mediante la interpretación de los datos estadísticos a través de dos tipos de indicadores temporales: la frecuencia absoluta (número de veces, unidades o casos en los que se verifica el uso de medios sociales) y la frecuencia relativa (proporciones o porcentajes). La interpretación de los resultados situados en el estudio del contexto laboral actual nos ayuda a establecer una investigación más completa y exhaustiva.

El estudio se ha llevado a cabo mediante encuestas realizadas a 20 periodistas profesionales que, en los periódicos locales de Sevilla, $A b c$ de Sevilla y Diario de Sevilla, asumen las labores propias del periodista digital. El 100\% de fiabilidad en los resultados obtenidos viene garantizado por el hecho de trabajar con todos los sujetos que componen la población objeto de estudio (no es necesario seleccionar 
una muestra), entendida como "el conjunto de todos los individuos (objetos, personas, eventos, etc.) en los que se desea estudiar el fenómeno", apunta Alvira Martín (2011: 7), quien explica que si recogemos la información de toda la población mediante un cuestionario estamos ante un censo y no ante una encuesta, si bien matiza que si atendemos a la identificación del método de la encuesta con la utilización de cuestionarios estructurados, los estudios censales también son encuestas. Esto no implica que la muestra censal que utilizamos no pueda ser tomada a su vez como muestra de lo que pueda hallarse entre la profesión periodística en otros medios de comunicación a nivel nacional español en un ejercicio de estadística inferencial.

El acceso a los encuestados se ha llevado a cabo a través de un cuestionario autoadministrado, enviado por correo electrónico al responsable de cada sección digital del periódico, quien lo distribuyó entre el resto de miembros del equipo. Una vez impresos y cumplimentados manualmente, el mismo responsable de la sección realizó la devolución, en un caso y en el otro, fueron enviados vía correo electrónico. Las principales ventajas de este método de envío se encuentra en la anulación del sesgo que produce la presencia del entrevistador; es un sistema económico de recogida de datos y la facilidad para que el encuestado responda en el momento que considera oportuno. La principal desventaja en nuestro caso es que no se posee la certeza absoluta sobre la influencia de terceras personas en las respuestas.

El diseño del cuestionario conlleva la distribución temática de las 25 preguntas de las que consta, excepto las preguntas introductorias sobre edad, sexo y rol profesional, de las que se extraen como resultados que los encuestados responden a unas edades comprendidas entre los 25 y 48 años de edad, siendo la media de edad la de un adulto joven (29 años). El sexo corresponde a 13 hombres y 7 mujeres y los roles que ocupan en la sección digital son: redactor (12), redactor web o digital (5), programador (1) y responsable de comunicación (1) y periodista digital (1).

La agrupación temática evita el desconcierto del entrevistado y cada bloque contiene preguntas introductorias. En primer lugar, se plantean los usos personales, después los profesionales, se pasa a continuación a las expectativas, siendo el centro del cuestionario, precisamente el uso profesional de los medios sociales. En cuanto a tipología, las respuestas son dicotómicas y múltiples en algunos casos; figuran alternas preguntas cerradas y de elección múltiple con opciones de respuesta en algunos casos excluyentes y en otros casos, inclusivas. Alguna cuestión también plantea respuestas con un ítem abierto para obtener información añadida en la que el encuestado puede añadir una respuesta que no está incluida entre las opciones propuestas. Las preguntas de estimación nos han servido para comprobar la regularidad y el alcance o importancia de un hecho o de una idea con respuestas graduadas en intensidad sobre el punto de información, más allá de la distribución de frecuencias de las respuestas emitidas. En último término, buscamos obtener una escala de valores entre las respuestas de los encuestados, si bien hemos huido de preguntas abiertas en la que se puede obtener mucha información, pero obligan a tener una gran complejidad en la codificación.

La exposición de los resultados se apoya en la lógica deductiva. Hemos intentando describir de forma comprensiva un conjunto de datos brutos desde lo más general a lo más concreto siguiendo la disposición de las preguntas del cuestionario. 
Como indica Salafranca, Sierra, Núñez y otros (2005: 163), "el objetivo del análisis de los datos es reducir y sintetizar la información para darle sentido. Y el objetivo de la interpretación es combinar los resultados del análisis con valores asumidos, criterios y patrones a fin de elaborar conclusiones, juicios y recomendaciones".

\section{ANÁLISIS DE RESULTADOS}

De las encuestas realizadas a los periodistas que desarrollan su labor en las secciones digitales de los periódicos Abc de Sevilla y Diario de Sevilla, se desprende el concepto de mayoritaria aprobación (60\%) de la actividad formativa sobre medios sociales que reciben los nuevos periodistas en las aulas universitarias, incluso un $40 \%$ cree que esta formación merece un notable. No obstante, estudios realizados sobre nivel formativo de los estudiantes de grados de periodismo en universidades públicas y privadas indican que no en todas ellas se imparten contenidos en asignaturas de Tecnología dirigidos a la formación en medios sociales ni en nuevos perfiles profesionales. En esto sí coinciden el 55\% de los profesionales que piensa que las universidades españolas no están reciclando suficientemente sus ciclos formativos en relación a las nuevas demandas profesionales, de lo cual se desprende un evidente autodidactismo, corroborado por el hecho de que sólo un $25 \%$ de los profesionales en ejercicio asevera haber seguido un curso para formarse como Community Manager o Social Media Editor parece confirmar este concepto. En casos afirmativos, Aerco y Lynda.com son los lugares elegidos.

Un $75 \%$ de los encuestados está de acuerdo $(65 \%)$ o totalmente de acuerdo (10\%), frente a un $25 \%$ que manifiesta su disconformidad con los nuevos perfiles profesionales que se están gestando con la incursión de los medios sociales, aunque ello suponga lazos con el concepto de 'periodista multiuso' vinculado a los nuevos perfiles, que es compartido por un $60 \%$ de los encuestados, de los cuales un $50 \%$ está de acuerdo y un $10 \%$ muy de acuerdo con ella, mientras que el $35 \%$ está en desacuerdo $(30 \%)$ o muy en desacuerdo $(5 \%)$.

Estas expectativas vienen de la mano del afianzamiento del uso profesional de la red, como lo atestigua el hecho de que un $65 \%$ dedique más de 6 horas a su uso para fines profesionales, seguido a notable distancia de un $25 \%$ que le consagra de 5 a 6 horas y un $10 \%$ que sólo emplea Internet 3 o 4 horas diariamente. Los motivos laborales son los principalmente señalados por los encuestados: llevar a cabo sus actividades de trabajo (75\%), seguir noticias de su interés $(45 \%)$ y cumplir sus labores académicas $(40 \%)$, incluso por encima de los motivos personales: mantener contacto con sus familiares y amigos (35\%) y hacer amistades (30\%).

Esta misma tendencia por el uso profesional de la red se extiende a las actividades desempeñadas, que resultan ser de naturaleza más pasiva y/o receptiva que activa y/o creativa (véase figura 1). El 95\% visita un sitio web de noticias, el $90 \%$ lee blogs, el $85 \%$ ve vídeos y el $80 \%$ descarga ficheros, frente a actividades que presenta una menor repercusión como subir un vídeo a un sitio web (40\%) y escribir regularmente en un blog (40\%). 
Fig. 1 Usos de lso periodistas profesionales de Internet

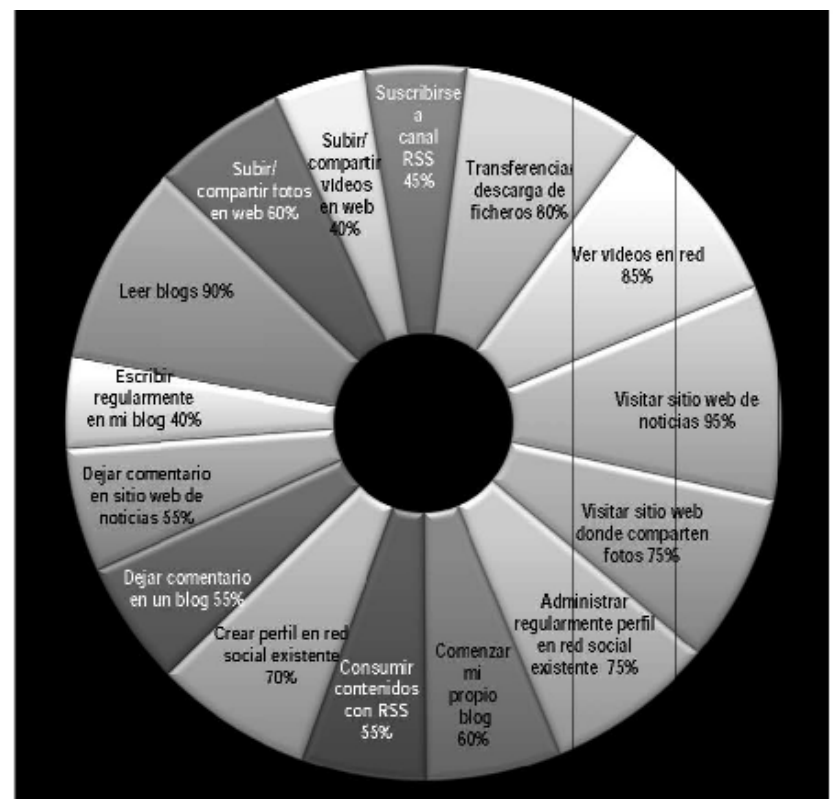

Fuente: Elaboración propia

Dentro de la web, los medios sociales representan una herramienta fundamental para el profesional del periodismo. Este dato no sólo es avalado por el hecho de que el $95 \%$ de los encuestados registren que se sirven de ellos para fines profesionales, también por el reconocimiento del $95 \%$ de los encuestados sobre su uso para herramienta de investigación profesional y del $80 \%$ para promoción de trabajos y, sobre todo, por el grado de significación de los medios, ya que un el $100 \%$ los considera herramientas importantes $(60 \%)$ o muy importantes $(40 \%)$ para el ejercicio de la profesión.

Unos de los medios sociales más destacados de la Web social o 2.0 son las denominadas redes sociales y el microbloggin, que se presentan ante el periodista como verdaderas arquitecturas de datos y contactos o, lo que es lo mismo, de información y comunicación (véase figura 2). Entre ellas, Facebook (90\%) es la red social más seguida, secundada por Twitter (85\%). Con un $55 \%$ se sitúan Tuenti y YouTube. Con menor alcance se encuentran Linkedin (35\%), Flickr (30\%), Foursquare (20\%) y Xing (5\%). Smallword.net, Bimeo y Blip.TV cuentan con la aceptación aislada de algún encuestado. 
Fig. 2 Medios sociales utilizados por los periodistas profesionales

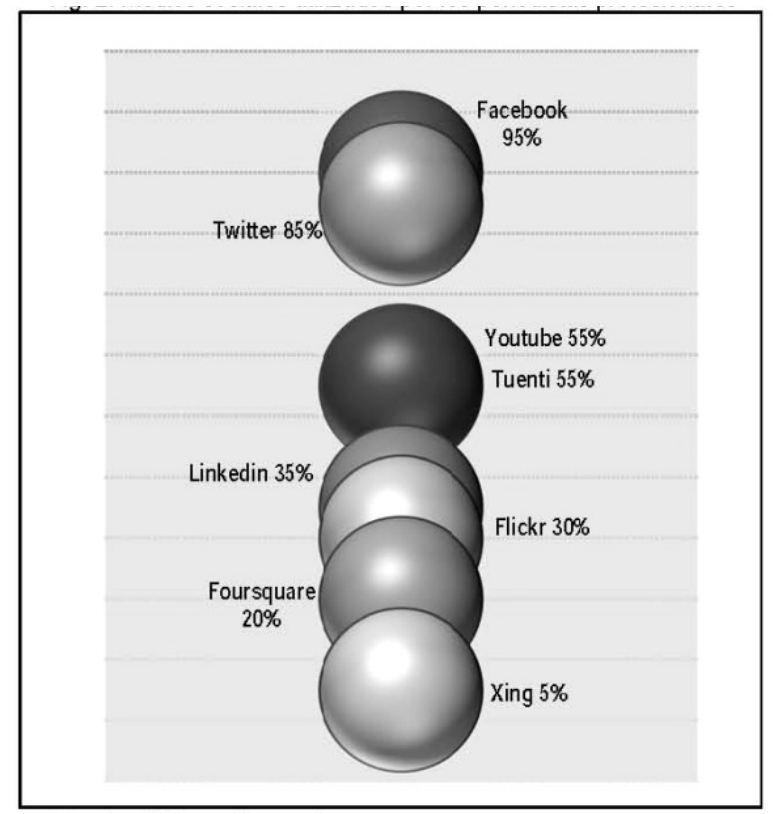

Fuente: Elaboración propia

El uso de estas redes sociales implica la creación y la administración de un perfil, tareas a las que se dedican un $70 \%$ y $75 \%$ de los encuestados que acceden a la red, respectivamente. Sólo el $10 \%$ manifiesta que tienen al menos un perfil en una red social, mientras que un $70 \%$ posee de dos a cuatro y un $20 \%$ más de cinco perfiles. En cuanto a frecuencia en la administración de dichos perfiles es, esencialmente, diaria (45\%) y semanal (35\%), frente a la anual (10\%) o la nula $(5 \%)$.

Los fines para los que se emplea un perfil en una red social son diversos dependiendo de la tarea y de la periodicidad (véase figura 3). Podemos decir, por tanto, que están claros sus usos, pero no así la frecuencia, propio de la libertad que permite la red. En el día a día, el periodista se dedica fundamentalmente a subir vídeos $(42,09 \%)$. Cada dos o tres días suele escuchar música $(32,62 \%)$, subir fotos a la red $(22,96 \%)$ y enviar mensajes a contactos $(21,75 \%)$. Un par de veces a la semana instala aplicaciones informáticas $(36,68 \%)$, envía mensajes a contactos $(20,84 \%)$ y escribe post $(20,84 \%)$. El hecho de subir fotos $(29,23 \%)$ y enviar mensajes a contactos $(20,76 \%)$ es una tarea programada cada dos o tres semanas, mientras que de manera esporádica también escribe post $(29,29 \%)$ y sube fotos a la red $(20,61 \%)$. Un $28,53 \%$ reconoce que nunca sube vídeos a la red y un $27,03 \%$ no envía mensajes a contactos. 
Fig. 3 Actividades realizadas por los periodistas profesionales en los medios sociales

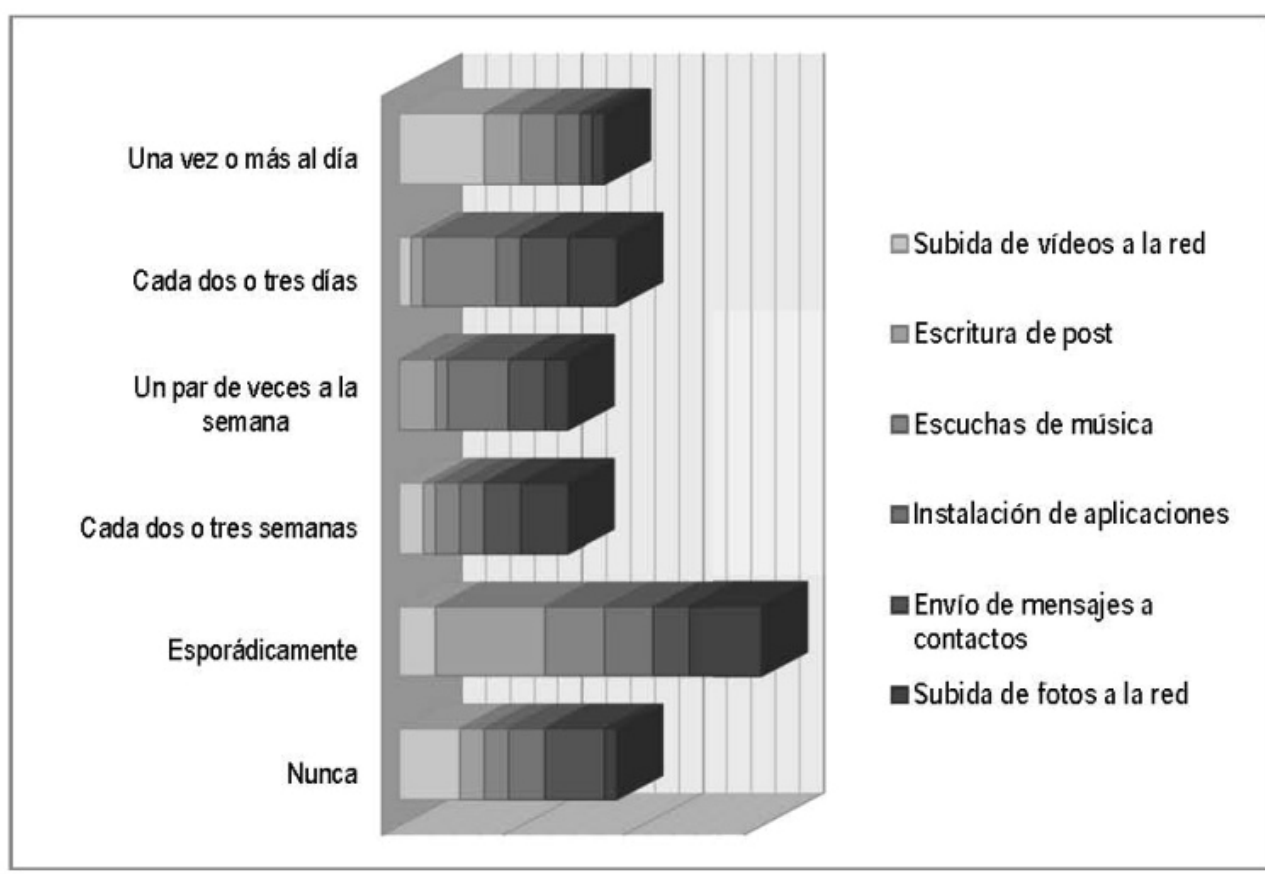

Fuente: Elaboración propia

Si nos fijamos en cada una de las actividades, se observa que la subida de vídeos a la red es seguida una vez o más al día por el $38,88 \%$, pero existe un $27,77 \%$ que reconoce que no lo hace nunca, frente al consenso que plantea la escritura de post. Es evidente que es una actividad de ejecución esporádica como lo respalda el $47,36 \%$, aunque existe un $15,78 \%$ que la hace una vez o más al día o un par de veces a la semana. Escuchar música es una actividad que se suele hacer cada dos o tres días $(31,57 \%)$ o esporádicamente $(26,31 \%)$; también existe un $15,78 \%$ que dice escuchar música una vez o más al día.

En cuanto a la instalación de aplicaciones se da mayor disparidad. Un $27,77 \%$ afirma que lo hace un par de veces a la semana y un $22,22 \%$ esporádicamente, si bien un $16,66 \%$ dice no hacerlo nunca y el resto de porcentajes se reparte entre el resto de opciones. También el envío de mensajes es una tarea donde no existe consenso. Está claro que la frecuencia menor es la de una vez o más al día (5,26\%). Un $26,31 \%$ afirma no enviar a través de la red mensajes a contactos, frente al $21,05 \%$ que reconoce hacerlo cada dos o tres días. Un 33,33\% de los encuestados reconoce que de forma esporádica sube fotos a la red, mientras que el otro 33,33\% se reparte entre los que lo hacen cada dos o tres semanas $(22,22 \%)$ o un par de veces a la semana $(11,11 \%)$. En la misma proporción son los que no lo hacen nunca o lo hacen una vez o más al día $(5,55 \%)$. 
Entre los principales usos que hacen de los medios sociales en su quehacer periodístico, destacan el hallazgo de material informativo complementario y la promoción mediática a los que los encuestados otorgan una importancia alta del 55,17\% $\mathrm{y}$ del $53,33 \%$, respectivamente (véase figura 4). El hallazgo de material informativo complementario es primordial, como lo recoge el hecho de que sólo un $6,89 \%$ le otorgue poca importancia, frente al $93,1 \%$ que lo valora con una importancia media o alta. Con menor rotundidad, la promoción mediática sigue el mismo esquema. Un $46,66 \%$ le otorga media o poca importancia, frente al $53,33 \%$ que le confiere una importancia alta. Menor diferencia encontramos en el contacto con otros colegas.

Para un 58,62\% tiene poca importancia y para el $41,36 \%$ la tiene media o alta a partes iguales. Tampoco es resaltable en importancia el control de la agenda informativa donde aproximadamente un tercio se acoge a cada una de las opciones en la línea de lo que ocurre con el logro de fuentes y testimonios, donde despunta ligeramente con un $40 \%$ la opción de poca importancia. Por su parte, la cesión de marca de identidad recibe media o poca importancia $(82,75 \%)$ frente al $17,24 \%$ que le confiere mucha importancia. Esta tendencia se mantiene en la obtención de documentos gráficos o audiovisuales, donde un $21,42 \%$ lo valora con una importancia alta, mientras que el $80,56 \%$ le da una relevancia media o baja y también en la creación de redes de contacto. Un $28,12 \%$ le proporciona mucha importancia y un $72,87 \%$ una importancia media o baja.

Fig. 4. Usos periodísticos de los profesionales en los medios sociales

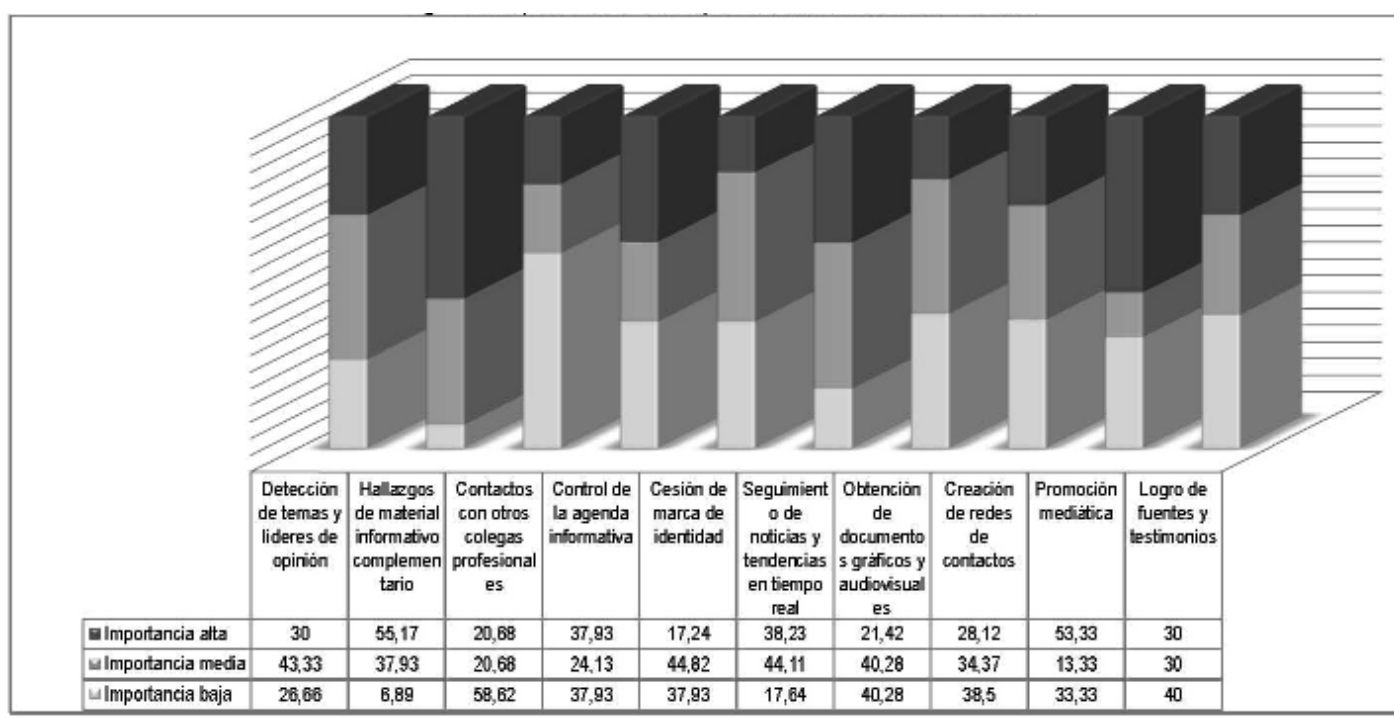

Fuente: Elaboración propia

Los resultados, en términos generales, sobre los principios más estimados por los periodistas en el uso de medios sociales indican que la arquitectura de identidad 
personal o virtual se encuentra entre los que se le otorga una relevancia baja $(30,30 \%)$, mientras que la adaptabilidad de usuarios para establecer contactos es de relevancia alta $(28,12 \%)$. En el baremo de relevancia media se encuentra, fundamentalmente, la ejecución del trabajo colaborativo $(29,16 \%)$ y la formación de inteligencia colectiva $(16,66 \%)$.

En un análisis más exhaustivo por principios, comprobamos que el más apreciado es la adaptabilidad de usuarios para contactos con un $87,5 \%$ proveniente de importancia alta $(56,25 \%)$ y media $(31,25 \%)$, frente al $12,5 \%$ de importancia baja (véase figura 5). Por su parte, la arquitectura de identidad personal o virtual es el criterio peor juzgado con un $71,42 \%$ de importancia baja, frente al $21,42 \%$ de importancia media y del $7,14 \%$ de importancia alta. El resto de principios son valorados de forma equitativa entre las tres opciones con una importancia media, muy asentada en la ejecución de trabajo colaborativo $(87,5 \%)$, la formación de inteligencia colectiva (50\%), la cooperación para resolver conflictos $(41,17 \%)$ y la movilización de redes sociales para servicio público con una media aproximada del 30-35\%. Las relaciones empáticas sin barreras se valoran en una importancia media-alta $(70,58 \%)$.

Fig. 5. Nivel de estimación de los periodistas sobre los principios de uso de los medios sociales

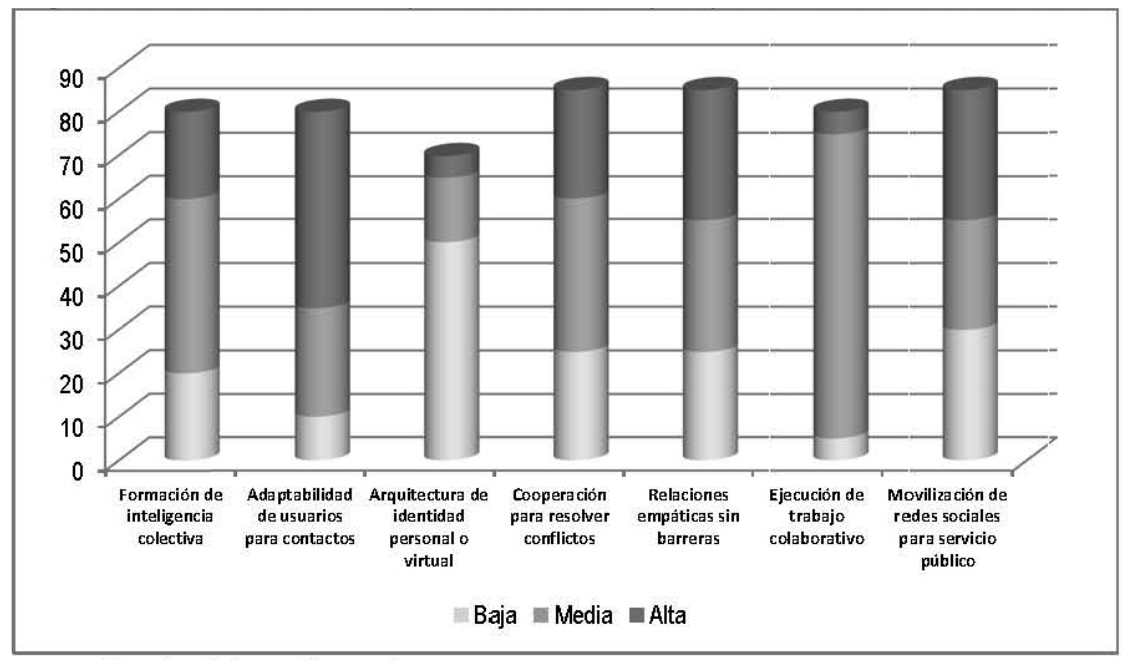

Fuente: Elaboración propia

Uno de los principios más valorados para el periodista es el grado de credibilidad que supone recoger o hacerse eco de datos textuales, testimoniales, gráficos o audiovisuales de la red. Si bien, sólo un 35\% reconoce haber utilizado alguna vez una imagen publicada por un medio social para formar parte de noticias o reportajes, hay que considerar que un $70 \%$ cree que la información a la que acceden a través de los medios sociales goza de un nivel de aprobado (40\%), notable $(25 \%)$ o sobresaliente (5\%), frente a un $30 \%$ que le otorga un suspenso. Este respaldo de con- 
fianza se revela también a través de alto porcentaje de encuestados que afirma conocer $(65 \%)$ o haber utilizado $(60 \%)$ aplicaciones en redes sociales que permiten agregar fuentes para coberturas en directo.

\section{CONCLUSIONES}

Entre las conclusiones del estudio encontramos las siguientes:

1. Los periodistas están de acuerdo con los perfiles profesionales gestados en la red, como prueba el $75 \%$ de los encuestados, de lo cual deriva la noción de que las nuevas figuras (community manager, social media editor, etc.) no representan una amenaza laboral para los periodistas en activo, sino la evolución propia de la profesión en su proceso de adaptación a las tecnologías digitales.

2. Existe un trasvase de uso personal, y por ende de lenguaje natural, del periodista en su relación con los medios sociales a la labor profesional, lo que se traduce en un alto nivel de dedicación de tiempo, teniendo en cuenta que el $80 \%$ de los encuestados le dedica entre 5 y 6 horas o más al día.

3. Los medios sociales son valorados como una herramienta de trabajo (el $95 \%$ de los encuestados se sirven de ellos para fines profesionales), avalado por el alto índice de credibilidad otorgada (70\%), pero la acción que desempeñan con ellos es claramente pasiva, más de recepción de datos (leer blogs, visitar sitios web de noticias, ver vídeos en red) que de creación de datos (subir vídeos en web, escribir en el blog).

4. Los principales usos dados por los periodistas locales son fundamentalmente dos: herramienta de investigación profesional y promoción de trabajos. Con respecto a la investigación profesional, los periodistas recurren a los medios sociales básicamente como fuentes de información compartidas, formando parte de la fase de hallazgos de material noticioso donde los datos de los medios sociales se utilizan como complemento a otros datos obtenidos por distintas vías. Con respecto a la promoción de trabajos, la difusión de material aparece más limitada a fotografías y vídeos que a textos noticiosos propiamente dichos.

5. En definitiva, los periodistas locales no introducen material informativo en los medios sociales, sino en sus propios medios de comunicación a los que reserva el proceso de redacción y creatividad. Aunque el $60 \%$ reconoce haber utilizado aplicaciones de redes sociales que permiten agregar fuentes en cobertura en directo, podemos afirmar que estos profesionales permanecen aún en una fase primaria que dista del potencial informativo de los medios sociales, explotado por periodistas de todo el mundo que han dado cobertura a grandes acontecimientos internacionales (por ejemplo, las revueltas de El Cairo de 2011). Hasta el momento los periodistas profesionales sólo ven en los medios sociales la oportunidad de hacerse presente en la realidad virtual con el fin de crear vínculos de interés para la obtención de información complementaria y no como un vehículo que permite cubrir acontecimientos de manera complementaria a la ofrecida en los medios de comunicación en los que trabajan y que les permite ampliar la conectividad emocional. 


\section{REFERENCIAS BIBLIOGRÁFICAS}

Alvira MarTín, F. (2011): La encuesta: una perspectiva general metodológica. Cuadernos Metodológicos, 35. Centro de Investigaciones Sociológicas, Madrid, $2^{\mathrm{a}}$ edición revisada.

APM A11 (2011): Informe anual de la profesión periodística 2011. Madrid.

BreIner, J. (2012): “¡Atrévete!”. Periodistas, 28, pp. 12-14.

CAstells, M. (ed.) (2006): La sociedad red: una visión global. Madrid: Alianza, p. 32 y 39.

GHannam, J. (2011): Social Media in the Arab World: Leading up to the Uprisings of 2011. Center for International Media Assistance, 3 de febrero de 2011. Disponible en: http://cima.ned.org/publications/social-media-arab-world-leading-uprisings-2011-0 [Consultado: el 15 de noviembre de 2011].

Herreros, P. (2012): "Entrevista a Ramón Puchades, Director de Redes Sociales de Unidad Editorial". Comunicación se llama el juego. Disponible en: http://comunicacionsellamaeljuego.com/entrevista-a-ramon-puchades-director-de-redes-sociales-de-unidad-editorial-y-ii/ [Consultado: 16 de junio de 2012].

LAJAS, J. (2011): "Al Jazeera usa la cobertura de las protestas en Egipto para promocionarse vía Twitter". PeriodismoCiudadano.com, 5 de febrero de 2011. Disponible en: http://www.periodismociudadano.com/2011/02/05/al-jazeera-usa-la-cobertura-de-lasprotestas-en-egipto-para-promocionarse-via-twitter/ [Consultado: 15 de noviembre de 2011].

LóPez, D. C. y Freire, M. (2010): "Las rutinas del periodista en el ambiente de convergencia: un estudio sobre la relación entre las fuentes de información y el uso de tecnologías en la radio". Actas del Congreso Internacional AE-IC Málaga 2010. Disponible en:

http://www.aeic2010malaga.org/esp/secciones_det.asp?id_seccio=7\&id_slot=16\&id_seccion $=5$ [Consultado: 10 de diciembre de 2011].

MACIÁ BARBER, C. (2007): "Un análisis de la utopía del periodismo ciudadano desde la perspectiva del reportaje interpretativo". Estudios sobre el Mensaje Periodístico, 13, pp. 123 144.

Meyer, P. (2007): "Prólogo" en BRIGGS, Mark: Periodismo 2.0. Una guía de alfabetización digital. Texas: Knight Centyer for Journalism in the Americas. University Of Texas At Austin.

Molina, M. (2012): “Nosotros también somos Silicon Valley”. Periodistas, 28, pp. 8-10.

OrinuElA, J. L. (2012): “Fuentes para los Juegos Olímpicos de Londres 2012”. eCuaderno. Disponible en: http://www.ecuaderno.com/2012/07/03/fuentes-para-los-juegos-olimpicos-de-londres-2012/ [Consultado: 4 de julio 2012].

Novosti, R. (2011): "Redes sociales adquieren protagonismo decisivo en revuelta de Egipto". Disponible en:

http://sp.rian.ru/science technology space/20110202/148292822.html [Consultado: 10 de octubre de 2011].

SalafrancA, L.; Sierra, V.; NúÑEz, I. et al. (2005): Análisis estadístico mediante aplicaciones informáticas. SPSS, Statgraphics, Minitab y Excel. Departament de Metodología de les Ciències del Comportament. Facultat de Psicología, Universidad de Barcelona.

SAn Martín, E. (2012): "Gotas de optimismo" en Periodistas, 28, pp.7.

SÁnchez Gonzales, H. M. (2012): "Los dispositivos móviles y la conectividad emocional con el usuario". TechPuntoCero. Disponible en:

http://www.techpuntocero.com/2012/05/15/los-dispositivos-moviles-y-la-conectividademocional-con-el-usuario [Consultado: 17 de mayo de 2012].

VARELA, J. (2005): "Periodismo 3.0, la socialización de la información”. Telos, 65, (octubrediciembre). Disponible en: 
http://sociedadinformacion.fundacion.telefonica.com/telos/articulocuaderno.asp@idarticulo $=7 \&$ rev $=65 . \mathrm{htm}$ [Consultado: 10 de diciembre de 2011].

VARGAS, E. (2010): ¿¿Por qué para El País de España las redes sociales son importantes?”. Clasesdeperiodismo.com. Disponible en:

http://www.clasesdeperiodismo.com/2010/10/04/\%C2\%BFpor-que-para-el-pais-deespana-las-redes-sociales-son-importantes/ [Consultado: 12 de diciembre de 2011].

ZAK, P. (2010): "Social Networking Affects Brains Like Falling in Love". Fast Company, 147, (julio/agosto). Disponible en: http://www.fastcompany.com [Consultado: 17 de noviembre de 2011].

\title{
RESUMEN
}

Este artículo pretende dar a conocer la existencia de un periodismo que no queda exento de las innovaciones que trae consigo la Web 2.0. Los medios están intentando adaptarse a las exigencia de la convergencia digital y de los medios sociales y los periodistas tienen que afrontar los nuevos retos profesionales como consecuencia de la revolución tecnológica, mediática y social. Se determina la existencia de los nuevos perfiles profesionales bajo demanda laboral y en el ejercicio de la profesión, el uso de las plataformas sociales por parte de los periodistas y la conectividad emocional que se establece con la audiencia en estos entornos. Utilizamos la encuesta como técnica metodológica cuantitativa y cualitativa para analizar a nivel local la opinión de los periodistas de dos diarios editados en Sevilla: Abc y Diario de Sevilla.

Palabras clave : Nuevos perfiles profesionales, periodismo digital, medios sociales, prensa de Sevilla

\begin{abstract}
This paper aims to shed light on a Journalism that does not remain exempt from the innovations that Web 2.0 brings inside. The media try to adapt to the needs of two hot topics: digital convergence and social means, and the journalists have to confront the new professional challenges as consequence of the technological, media and social revolution. It is determined the existence of the new professional profiles upon labor demand and in the professional exercise, the use of the social platforms by the journalists and the emotional connectivity that is established by the readership in these environments. We employ the poll as quantitative and qualitative methodological technology to analyze at local level the opinion of the journalists of two newspapers edited in Seville: Abc and Diario de Sevilla.
\end{abstract}

Keywords: New professional profiles, digital Journalism, social means, press of Seville

\section{RÉSUMÉ}

Cet article réflechit sur les changements que la Web 2.0 implique pour le journalisme. Les mèdia essaient de s'adapter aux nouveaux défis professionnaux que la revolution technologique et sociale produisent. Des nouveaux profils proféssionels sont demandés pour le secteur, et la connectivité emotionnelle est un trait necéssaire pour communiquer avec les lecteurs. Dans la pièce s'utilisent des études quantitatifs et qualitatifs pour analiser l'opinion des journalists de deux journals en Seville, $A b c$ et Diario de Sevilla

Mots clé: nouveaux profils professionaux, journalisme digital, media sociaux, presse en Seville 